\title{
¿Cómo se está formando al cuerpo docente centroamericano para enseñar la lectoescritura inicial? Aportes de una investigación regional a partir de cinco estudios de caso
}

How are Central American teachers being prepared to teach early grade literacy? Regional research findings from five case studies

\section{Volumen 20, Número 2 \\ Mayo - Agosto \\ pp. 1-33}

\author{
Paola Andrade-Calderón \\ Rebecca Stone \\ Josefina Vijil
}




\title{
¿Cómo se está formando al cuerpo docente centroamericano para enseñar la lectoescritura inicial? Aportes de una investigación regional a partir de cinco estudios de caso
}

How are Central American teachers being prepared to teach early grade literacy? Regional research findings from five case studies

\author{
Paola Andrade-Calderón ${ }^{1}$ \\ Rebecca Stone ${ }^{2}$ \\ Josefina Vijil ${ }^{3}$
}

\begin{abstract}
Resumen: Una de las principales limitaciones para el avance educativo en los países de Centroamérica es la insuficiente formación de sus docentes. Por lo general, los programas de desarrollo profesional dejan grandes vacíos respecto al dominio de los contenidos a enseñar, de los conocimientos pedagógicos sobre cómo enseñar y de las habilidades para una mediación docente pertinente. Asimismo, es frecuente que la educación del magisterio se diseñe al margen de los planes del sistema escolar y de la formulación de las políticas educativas. En este artículo se discute esta problemática y se explica el marco conceptual, metodología y desarrollo de la investigación que analizó la formación inicial docente (FID), en el ámbito de la Lectoescritura inicial (LEI). El estudio, de corte cualitativo, se desarrolló en instituciones educativas de Guatemala, El Salvador, Honduras, Nicaragua y Costa Rica. Se recolectaron y triangularon datos de tres fuentes: (1) mapas curriculares de la FID en el ámbito de la LEl, organizados a partir de un marco conceptual que consideró la evidencia investigativa más reciente sobre el aprendizaje de la lectura y escritura; (2) entrevistas a docentes formadores y a docentes en formación; y (3) observaciones de sesiones de clase en aulas universitarias y en un centro normalista donde transcurre la formación docente. La información recolectada permitió analizar las características e implementación de los programas de la FID e identificar sus fortalezas y brechas. Los resultados indican que el cuerpo docente que enseñará la LEI en Centroamérica no está recibiendo la formación que los prepare para tan importante tarea y que los programas de FID disponibles no se basan en la evidencia actual sobre cómo se aprende la LEI. Los hallazgos han sido formulados para promover la discusión, en las instituciones participantes, sobre los ajustes que sus programas deberían experimentar para acercar la formación del magisterio en la enseñanza de la LEI a lo que sugiere la evidencia científica. Se ofrecen además reflexiones para motivar la discusión sobre las políticas de FID a nivel nacional y regional.
\end{abstract}

Palabras clave: investigación curricular, lectoescritura inicial, formación docente, alfabetización.

\footnotetext{
1 Coordinadora de Investigación RedLEI, Guatemala. ORCID: https://orcid.org/0000-0002-8688-8797 Dirección electrónica: paandrade@uvg.edu.gt

2 Directora del Programa de Capacidades LAC Reads, Estados Unidos. Especialista en lectoescritura inicial e investigadora de American Institutes for Research, Estados Unidos. ORCID: https://orcid.org/0000-0003-1201-6205 Dirección electrónica: rstone@air.org

3 Doctora en Ciencias de la Educación por la Universidad Católica de Lovaina, Bélgica. Especialista en lectura temprana del Programa de Capacidades LAC Reads, Estados Unidos. ORCID: https://orcid.org/0000-0003-1354-2485 Dirección electrónica: jviji@juarezassociates.com
}

Artículo recibido: 15 de noviembre, 2019

Enviado a corrección: 31 de marzo, 2020

Aprobado: 20 de abril, 2020 


\begin{abstract}
The main obstacle to educational progress in Central America is the insufficient training of their teachers. In general, teacher education programs do not help teachers achieve sufficient mastery of content they will be expected to teach, pedagogical knowledge on how to teach that content in the classroom, and skill development; they are also isolated from the school system and the formulation of educational policies. This article discusses this challenge and explains the conceptual framework, methodology and development of the research that analyzed preservice teacher training 4 in the field of initial literacy (EGL)5. A qualitative research methodology was used to conduct the study in Guatemala, El Salvador, Honduras, Nicaragua and Costa Rica. The preservice teacher education analysis triangulated information obtained from the following sources: (1) a mapping of the preservice literacy curricula against an evidence-based EGL conceptual framework, (2) interviews with preservice teacher trainers and their students, and (3) observations of preservice teacher training classes. The research provides data about the characteristics of preservice teacher education programs and their implementation, which allows for an analysis of their strengths and gaps. The results indicate that the teachers who will teach EGL in Central America are not receiving the training that prepares them for such an important task and that the preservice programs available are not based on current evidence on EGL learning. The findings have been formulated to promote discussion, in participating institutions, about the adjustments that their programs should undergo to bring EGL teacher training closer to what the scientific evidence recommends. Reflections are also offered to motivate discussion on preservice teacher education policies at the national and regional levels.
\end{abstract}

Key words: curriculum research, early grade literacy, teacher education, literacy.

\title{
1. Introducción
}

Las evaluaciones del aprendizaje escolar de los últimos años (Segundo y Tercer Estudio Regional Comparativo y Explicativo [SERCE y TERCE] y la Prueba Diagnóstica de Lectura Inicial [EGRA]) (Gove et al., 2015; UNESCO, 2016) revelaron que la niñez de Centroamérica (CA) no ha logrado los niveles deseados en el desarrollo de habilidades fundamentales como la lectura y la escritura (CIEN y Diálogo Interamericano, 2015). Los bajos resultados en estas materias se relacionan con la deficiente calidad de su instrucción (UNESCO, 2016), un aspecto estrechamente vinculado a la formación docente.

La investigación señala que el escaso conocimiento pedagógico afecta la práctica docente, debilita el aprendizaje y reduce el logro educativo del estudiantado (Akyeampong, Pryor y Ampiah 2006; Dembélé y Lefoka, 2007; Moon, Leach y Stevens, 2005; Pontefract y Hardman 2005; UNESCO 2016; Fondo de las Naciones Unidas para la Infancia [UNICEF], 2012). Ciertamente, la formación docente es central para mejorar el aprendizaje de la Lectoescritura Inicial (LEI), puesto que la calidad de la instrucción depende del conocimiento que se tenga de este ámbito y de cómo enseñarla (Chesterfield y Abreu-Combs, 2011; Cunningham, Perry, Stanovich, K. y Stanovich, P., 2004; McEwan, 2014; Moats, 2009; Rivkin, Hanushek y Kain, 2005).

\footnotetext{
${ }^{4}$ Preservice teacher training: Content - knowledge and skills - whose mastery is prescribed in teacher training curricula as a requirement for qualification as an elementary school teacher.

${ }^{5}$ Early grade literacy: The first and second stages of formal learning of reading and writing, which take place during childhood around the third grade of elementary school, and include the development of skills ranging from decoding to fluency and reading comprehension (Chall, 1983). Implicit in the concept is the theory that literacy acquisition results from intentional instruction in a school context.
} 
La literatura sobre el dominio que el cuerpo docente centroamericano tiene de las materias que se enseñan es escasa. De los pocos estudios existentes se sabe que hay una discrepancia preocupante entre los títulos académicos obtenidos y las competencias desarrolladas (Teacher Education and Development Study in Mathematics [TEDS-M], 2008). Además, se sabe que en muchos casos la Formación Inicial Docente (FID) es insuficiente para las demandas de calidad educativa. Por un lado, el alcance de los programas de formación se reduce a una comprensión limitada del currículo y sus didácticas, y a contenidos pedagógicos generales que no preparan para el uso de metodologías efectivas en la enseñanza de la LEI (Kyeyune et al., 2011; Louzano y Moriconi 2014; Oficina Regional de Educación para América Latina y el Caribe [OREALC] y Organización de las Naciones Unidas para la Educación, la Ciencia y la Cultura [UNESCO], 2013). Por el otro, los programas omiten contenidos importantes, como la enseñanza en contextos multilingües, un tema que demanda mucha atención en la región centroamericana (Akyeampong, Pryor, Westbrook y Lussier 2011).

La calidad de la formación docente pre-servicio en Centroamérica también es un tema poco estudiado, y se adolece de datos para hacer comparaciones significativas entre países. Algunos estudios cualitativos reiteran lo que ya se ha dicho al señalar sistemáticamente que, además de no proporcionar un grado suficiente de dominio de los contenidos ni conocimientos de metodologías centradas en el alumnado, la formación docente está poco vinculada al sistema escolar y a la formulación de políticas de educación, y solo incluye al final de la carrera, cuando ocurre, experiencias concretas de aplicación de lo aprendido al trabajo en el aula (UNESCO, 2012, pp. 44-45).

La falta de investigación sobre la Formación Inicial Docente (FID) deja a la región sin evidencia que guíe el diseño de planes de estudio para la preparación del futuro profesorado en la instrucción efectiva de la LEI (Bruns y Luque, 2015; LAC Reads Capacity Program [LRCP], 2016). Resulta necesario ampliar el conocimiento del contenido e implementación de los planes con los que se está formando a potenciales docentes en Centroamérica sobre lectoescritura inicial, para mejorar la instrucción en esta materia clave para el aprendizaje a lo largo de la vida. La Red para la Lectoescritura Inicial de Centroamérica y el Caribe (RedLEI) eligió la FID como el objeto de estudio de su primera investigación regional para contribuir a llenar este vacío, y a formular propuestas centradas en la búsqueda de la calidad educativa en los primeros grados. La investigación analizó la formación, en el ámbito de la LEI, del alumnado de magisterio en cinco instituciones educativas de los países en los 
cuales la Red se estableció inicialmente: Guatemala, El Salvador, Honduras, Nicaragua y Costa Rica. Las instituciones participantes son tanto del sector público como privado.

En el siguiente apartado se describen el marco conceptual y la metodología utilizados para analizar los programas de la FID en las instituciones participantes y responder a la pregunta de investigación: ¿Qué formación inicial recibe el cuerpo docente que enseña la LEI en Centroamérica? La RedLEI espera que los resultados del estudio se aprovechen para reflexionar sobre los cambios que requieren los programas de formación docente inicial, las políticas curriculares y la formación continua de educadores, tanto a nivel nacional como regional, para adecuarse a las necesidades y expectativas de calidad educativa de los países de la región.

\section{Referente Teórico}

El cuerpo docente es el pilar fundamental para el aprendizaje del estudiantado, y la calidad de su formación es el factor de mayor importancia para catalizar su potencial en la instrucción de la LEI. La formación docente de calidad implica el conocimiento de cómo se adquiere la LEI -los procesos cognitivos, sociales y afectivos que apoyan su desarrollo. La instrucción reflexiva y el diseño de estrategias efectivas para enseñar a leer y escribir se basan en el conocimiento tanto de la teoría de la LEI como de su aplicación en el diseño de planes de estudio adaptados a las necesidades del alumnado (Litt, Martin y Place, 2015).

Teniendo lo anterior como premisas, la RedLEI se propuso explorar la amplia literatura científica que informa sobre los conocimientos específicos o saberes que el cuerpo docente debe dominar, y su aplicación en el saber hacer la instrucción de la lectoescritura inicial como conjunto de habilidades complejas. La revisión de la literatura derivó en un marco conceptual de tres dimensiones del conocimiento teórico y práctico para la enseñanza efectiva en esta materia: Desarrollo Infantil; Conocimiento de la Lectoescritura y Evaluación de los Aprendizajes. Las dimensiones identificadas se explican a continuación.

\subsection{Dimensión 1. Desarrollo Infantil}

La comprensión de los procesos evolutivos que ocurren durante la niñez es fundamental para informar al docente de las necesidades de sus estudiantes en las diferentes etapas que experimenta al inicio de la vida escolar (Darling-Hammond y Bransford, 2005; Copple y Bredekamp, 2009). También es clave la comprensión de que el desarrollo infantil es el resultado de la influencia recíproca de factores biológicos, sociales, culturales y 
del contexto físico. Asimismo, se requiere conocer cómo ocurre el aprendizaje -como proceso mental y como práctica social- para incidir adecuadamente en la adquisición de la LEI. La literatura sugiere diez ámbitos específicos de conocimiento o saberes clave para alcanzar los niveles adecuados de formación docente en esta Dimensión.

\subsubsection{Teorías del desarrollo}

Es el conocimiento base para explicar el proceso de desarrollo como resultado de la influencia recíproca de las características biológicas y el contexto físico, social y cultural. Contribuye a determinar cómo influir adecuadamente en el proceso alfabetizador y a diseñar acciones efectivas aprovechando los procesos que lectores y escritores realizan para aprender la cultura escrita en varias etapas de la vida (Copple y Bredekamp, 2009; DarlingHammond y Bransford, 2005; Díaz, Villalón y Adlerstein, 2015; Bezerman y Kress, 2010 y Manghi, 2011).

\subsubsection{Teorías del aprendizaje y la motivación}

De acuerdo a la International Literacy Association (ILA, 2010), la comprensión profunda del desarrollo del lenguaje y su relación con la lectura son fundamentales para el dominio de las teorías del aprendizaje y la motivación que orientan al cuerpo docente en la toma de decisiones pedagógicas.

\subsubsection{Desarrollo del lenguaje}

Es el conocimiento de aspectos centrales del desarrollo, estructuras y estrategias del lenguaje con el que cuenta el magisterio para guiarse en la instrucción efectiva de la LEI (Adger, Snow y Christian, 2018; Hoyle y Adger, 1998; Menyuk y Brisk, 2005; Wong-Fillmore y Snow, 2000). Este conocimiento favorece la identificación de problemas en el desarrollo del lenguaje, tanto los que se resolverán con el tiempo como los que demandan intervención inmediata.

\subsubsection{Fundamentos de la enseñanza-aprendizaje de la lectura}

Es preciso que el cuerpo docente comprenda las etapas que conlleva el desarrollo de la lectura inicial, las habilidades a desarrollar en cada una de ellas, y la relación existente entre los componentes de nivel superior e inferior involucrados en el proceso (Hoover y Gough, 1990). 


\subsubsection{Fundamentos de la enseñanza-aprendizaje de la escritura}

El cuerpo docente requiere conocer las etapas por las que transcurre la niñez en la adquisición de la escritura y sus componentes, la grafía y el trazo, para el desarrollo posterior de la expresión escrita. Debe conocer que escribir bien demanda el dominio de varios niveles de conocimiento lingüístico: fonológico, morfosintáctico, léxico, pragmático y discursivo (Alamargot y Chanquoy, 2001) con el objetivo último de ayudar al alumnado a ser competente en el uso del lenguaje (Berman, 2004).

\subsubsection{Neurociencia de la Lectoescritura Inicial}

El trabajo docente requiere conocimiento sobre cómo aprende a leer y escribir el cerebro, y cómo se "reutilizan" las regiones cerebrales que fueron diseñadas biológicamente para otros fines para crear nuevos circuitos y conexiones en sintonía con el lenguaje escrito. Este conocimiento es clave para comprender que la eficiencia de las conexiones neurales recién formadas depende de las experiencias en los primeros años de vida y garantiza el éxito del proceso (Brem et al., 2010; Casey, Davidson, y Rosen, 2002; Diamond, 1996; Goldman-Rakic, 1987; Leppänen et al., 2010; Liston, et al. 2005; McLaren, Ries, Xu, y Johnson, 2012; Munakata y Yerys, 2001; Olesen, Westerber y Klingberg, 2004).

\subsubsection{Psicología y sociología de la lectoescritura}

Es la comprensión de que las personas piensan, aprenden y se desarrollan en contextos sociales utilizando el lenguaje como herramienta (Bruning y Kauffman, 2015). Esos contextos dan significado al aprendizaje de la escritura, la lectura, el género, el contenido y la audiencia (Bazerman, Farmer, Halasek y Williams, 2005) y dan forma a las trayectorias del estudiantado en el aprendizaje de la lectoescritura, incluso cuando no es explícitamente reconocido por el cuerpo docente (Barletta Manjarres, Cortez Roman y Medzerian, 2012).

\subsubsection{Impacto del contexto en el aprendizaje de la LEI}

La formación efectiva demanda el dominio de herramientas para conocer al estudiantado, a su comunidad y a sus prácticas letradas a fin de ofrecer una mediación docente ajustada a la forma particular en que viven en el mundo (Ferreiro y Teberosky, 1979; Teberosky, 2000) y a sus tradiciones lingüísticas (Lucas y Grinberg, 2008). 


\subsubsection{Bilingüismo}

La investigación ha documentado los beneficios del bilingüismo (Bialystok, 2011; Ortega, 2008; Romaine, 1995) y la efectividad de programas bilingües y de inmersión en dos idiomas en los que el alumnado aprende y desarrolla tanto el primero como el segundo, experimentando ventajas académicas y lingüísticas de largo plazo (Greene, 1999; G. McField y D. McField, 2014; Rolstad, Mahoney y Glass, 2005). La evidencia sugiere que el desarrollo del primer idioma apoya el aprendizaje del segundo (August y Shanahan, 2006; Genesee, Lindholm-Leary, Saunders y Christian, 2006). En los países de Centroamérica, donde se hablan varios idiomas además del español, el conocimiento sobre bilingüismo es especialmente importante para el docente alfabetizador pues un número importante del alumnado inicia la escuela con dominio de un idioma materno diferente al de instrucción. Las escuelas y sus docentes deben saber cómo ayudar a todos sus estudiantes a transferir las habilidades de la lengua materna a otra, y garantizar el éxito de los procesos de lectoescritura en L1 y L2.

\subsubsection{Trastornos del desarrollo de la lectura y escritura}

Este conocimiento describe y explica los retrasos en el aprendizaje de la lectura y la escritura respecto a las edades consideradas oportunas, y es fundamental para reconocer a tiempo estas dificultades (American Psychiatric Association, 2014). De acuerdo con Tunmer y Hoover (2019), las carencias de la niñez en la adquisición de la lectoescritura inicial podrían estar asociadas a problemas de decodificación o de comprensión del lenguaje. EI cuerpo docente alfabetizador, además de reconocer estos dos tipos de dificultades, debe conocer cómo ayudar al alumnado a superarlas. La formación docente debe además capacitar para que la enseñanza de la LEI se adecúe al contexto y necesidades particulares de la niñez.

\subsection{Dimensión 2. Conocimiento de la Lectoescritura}

La instrucción de la lectura y escritura inicial es mucho más compleja de lo que podría parecer a un observador casual. El cuerpo docente alfabetizador será efectivo en la medida en que comprende cómo el alumnado aprende a leer y escribir y qué factores facilitan el proceso. Debe, por tanto, estar preparado para ayudar a sus estudiantes a interpretar ideas complejas, analizar argumentos críticamente, sintetizar información de múltiples fuentes y usar la lectura para construir su conocimiento (National Research Council, 2010). Los once 
ámbitos o saberes de esta dimensión de la formación se relacionan con el conocimiento teórico sobre las habilidades para leer y escribir y el conocimiento práctico sobre cómo desarrollarlas, y preparan al cuerpo docente en el diseño y uso de metodologías para la instrucción efectiva de la LEI

\subsubsection{Lenguaje oral}

Sobre el lenguaje oral se asienta el desarrollo de la LEI (American Speech-LanguageHearing Association $[\mathrm{ASHA}], 2008)$. El uso del lenguaje oral durante la niñez modela la capacidad de expresión que, a la vez, favorece las habilidades internas sobre las cuales se establecerá la comprensión lectora y la expresión escrita. En consecuencia, la comprensión del lenguaje oral y su desarrollo es un aspecto clave para la instrucción de la lectoescritura (Stahl, Flanigan y McKenna, 2009).

\subsubsection{Concepto de lo impreso}

Los conocimientos básicos sobre cómo funcionan los materiales impresos y las funciones que cumplen -lectura de izquierda a derecha y de arriba hacia abajo; diferencia entre dibujos y letras; y que las letras y palabras transmiten mensajes- también constituyen un conocimiento fundamental para quien enseña a leer y escribir (Holdgreve-Resendez, 2010).

\subsubsection{Conciencia fonológica}

Es el conocimiento de que nuestro lenguaje está compuesto de unidades sencillas como los sonidos y que éstos pueden manipularse. El desarrollo de la conciencia fonológica y fonética es uno de los mejores "predictores de escolarización y de cuán bien los niños y las niñas aprenderán a leer durante los primeros dos años de instrucción" (National Reading Panel [NRP], National Institute of Child Health, \& Human Development, 2000 p.7). La comprensión de este concepto, por tanto, es necesaria para la instrucción efectiva de la LEI.

\subsubsection{Principio alfabético}

Es la comprensión de que existen relaciones sistemáticas y predecibles entre los sonidos y las letras. El reconocimiento de las letras no es natural para la niñez, sino que depende de una instrucción sistemática e intencional en la escuela. Se traduce en una 
habilidad que se automatiza cuando se utiliza la memoria de trabajo en la comprensión de lo que se lee (National Institute of Child Health and Human Development [NICHD], 2000).

\subsubsection{Vocabulario}

Es la comprensión de cómo se da significado a las palabras. El conocimiento de cómo las personas construyen su vocabulario constituye un aspecto fundamental para analizar el funcionamiento cognitivo de la comprensión lectora (NICHD, 2000).

\subsubsection{Fluidez lectora}

Es la comprensión de cómo se forman las capacidades para leer un texto con precisión, velocidad y entonación adecuada. La fluidez se logra cuando se ha automatizado la decodificación. Según la investigación, la automatización depende del desarrollo de la habilidad de reconocer las palabras y es un componente crítico para la comprensión lectora (NICHD, 2000).

\subsubsection{Comprensión lectora}

Es el conocimiento de cómo se construye la capacidad de dar significado y comprender un texto. El concepto define la finalidad última del aprendizaje de la lectura que resulta de la aplicación de estrategias para recordar, pensar, reflexionar, criticar y emitir opiniones. Las estrategias de comprensión lectora se enseñan de manera sistemática e intencionada desde el nacimiento y continúan a lo largo de toda la escolaridad (August y Shanahan, 2006; Camargo, Montenegro, Maldonado y Magzul, 2013; NICHD, 2000).

\subsubsection{Conexiones entre lectura y escritura}

Es el conocimiento de cómo los procesos de lectura y escritura se conectan y soportan mutuamente en apoyo a la comprensión (Graham y Hebert, 2010; International Reading Association, 2007; Shanahan, 2015). El lenguaje escrito no es un código de transcripción de unidades sonoras sino un sistema de representación gráfica del lenguaje hablado que simboliza enunciados lingüísticos y guarda relación con lo oral, aunque tiene propiedades específicas que van más allá de la simple correspondencia con los sonidos (Ferreiro y Gómez, 1986). 


\subsubsection{Grafomotricidad}

Consiste en la comprensión y reconocimiento de los patrones motores que derivan en la aptitud para el desarrollo y ejecución del trazo. La grafomotricidad es una habilidad que comienza con la macro motricidad (desplazamiento del cuerpo en el espacio), continúa con la motricidad media (movimiento del cuerpo y de los miembros sin cambiar de lugar) y termina con la motricidad final (rotaciones de las manos, digitaciones) (Alviz, 2012).

\subsubsection{Expresión escrita}

Es la comprensión de la capacidad de expresar ideas y conocimientos por escrito como actividad inteligente que depende de la activación de tres procesos de tipo cognitivo, interactivo y recursivo: la planificación, la transcripción y la revisión (Hayes, 1996; Hayes y Flower, 1980). Estos procesos se controlan y regulan mediante un mecanismo de monitoreo. El monitor simboliza la conciencia meta-cognitiva del escritor (Sitko, 1998) que ejerce una función auto-reguladora de la escritura, y supone conocimiento y control de las operaciones anteriores (Gallego, García, y Rodríguez, 2014). Escribir un texto requiere, por tanto, del dominio progresivo de una serie de operaciones intelectuales complejas (Condemarin y Medina, 2000).

\subsubsection{Convenciones de la escritura}

Es la comprensión de la habilidad de leer y escribir con relación a la sintaxis y la ortografía. El proceso sintáctico explica cómo están relacionadas las palabras y cómo se estructura la gramática del lenguaje. El ortográfico informa las reglas de la escritura, y el deletreo y puntuación correcta de las palabras (Berninger, y Amtmann, 2003; Bui, Schumaker, y Deshler, 2006; Englert et al. 1995; Witte y Faigley, 1981).

\subsection{Dimensión 3. Evaluación}

La comprensión teórica y práctica de la LEI prepara al cuerpo docente para seleccionar recursos apropiados para su instrucción (Adger, Snow y Christian, 2018). Debido a que las decisiones docentes tienen enormes consecuencias para la actividad estudiantil, es fundamental también la formación en el diseño y aplicación de instrumentos de medición y seguimiento del aprendizaje, especialmente en el uso de sus resultados para la toma de decisiones que mejoren la enseñanza. La tercera Dimensión de este marco conceptual incluye cuatro ámbitos de conocimientos y habilidades que, de acuerdo a la evidencia 
investigativa, capacitan al futuro docente para evaluar la adquisición de la lectoescritura inicial.

\subsubsection{Propósito y tipos de evaluación}

Es el conocimiento de que la evaluación es parte integral del proceso de enseñanza y aprendizaje y potencia su carácter dinámico e interactivo. La evaluación debe ser, por esencia, plural o multidimensional, para adecuarse a la complejidad del desarrollo del lenguaje escrito y a la heterogeneidad del alumnado. El cuerpo docente requiere dominio de un amplio repertorio de procedimientos, técnicas e instrumentos de evaluación, más allá de los que se utilizan tradicionalmente (Condemarin y Medina, 2000).

\subsubsection{Diseño de evaluaciones formativas}

Es la capacidad de elaborar herramientas de evaluación para la detección, el diagnóstico, el seguimiento del progreso (evaluación formativa) y la medición de los resultados (evaluación sumativa) (Condemarin y Medina, 2000) de cada una de las habilidades de la lectoescritura inicial que fueron detalladas en la Dimensión 2. El dominio de estas herramientas es fundamental para la autorregulación del aprendizaje y la enseñanza.

\subsubsection{Aplicación de evaluaciones formativas}

El cuerpo docente requiere conocimiento de los estándares educativos existentes y su aplicación al ámbito de la LEI. Requiere además dominio de una variedad de herramientas y métodos para planificar y evaluar el aprendizaje de la lectoescritura inicial tales como el monitoreo sistemático del rendimiento individual del alumnado, y la gestión del aula, de la escuela y del sistema (ILA, 2010).

\subsubsection{Interpretación y uso de los resultados de la evaluación}

Es el conocimiento que prepara para el uso e interpretación de los resultados de diferentes tipos de evaluaciones, mediante las que el cuerpo docente obtiene la información necesaria para adecuar la instrucción rápidamente. La aplicación de este conocimiento se da mientras la enseñanza está en progreso y contribuye también a que el estudiantado use sus resultados para ajustar y mejorar su propio aprendizaje (S. Chappuis y J. Chappuis, 2008).

El marco conceptual descrito se utilizó como referente para analizar la información recolectada en el marco de esta investigación obtenida a través del mapeo de los cursos de 
formación docente, entrevistas a educadores y educandos, y observaciones de aula. La información recolectada y analizada constituye evidencia del estado actual de la formación docente inicial para la lectoescritura en cinco instituciones de la región Centroamericana.

\section{Metodología}

En todas las instituciones participantes en esta investigación se aplicó una metodología y enfoque común que se expresa en un marco conceptual para guiar el análisis de la FID en lectoescritura inicial y técnicas de recolección y análisis de datos. Dado que cada institución constituyó una unidad de análisis propia, el informe de resultados se presenta en diferentes artículos a lo largo de este número monográfico. Cada artículo informa de la implementación de esta metodología común en un programa de formación docente, y propone hallazgos y recomendaciones que consideran el contexto del país y de la institución en el cual este programa se desarrolla. Los detalles sobre la implementación de la investigación y la descripción de las unidades de análisis de cada país, pueden consultarse en los artículos que conforman este número y abordan los resultados según el contexto particular en donde se desarrolló la investigación.

\subsection{Enfoque}

La RedLEl se planteó realizar una investigación regional para analizar la formación inicial docente en la LEI en instituciones del sector público y privado, como medio para incidir en la mejora del diseño de políticas y programas de educación magisterial en los países de Centroamérica. Los hallazgos se han traducido en recomendaciones sobre cómo alinear lo que la literatura sugiere que el futuro cuerpo docente debe comprender y saber hacer sobre la instrucción de la LEI, y lo que actualmente se les enseña. La investigación se propuso responder las siguientes preguntas:

1. ¿Cómo se alinea el currículo de formación inicial docente con la evidencia sobre cómo se aprende la lectoescritura en los primeros años y qué deben saber y poder hacer las docentes y los docentes para una instrucción efectiva?

2. ¿Cuáles son las fortalezas y las brechas del plan de estudios de formación inicial docente en relación con la evidencia investigativa?

3. ¿En qué se diferencia el currículum prescrito del currículo realmente aplicado en los programas de formación inicial docente en el ámbito de la lectoescritura inicial? 
Para encontrar respuestas a estas preguntas se diseñó un estudio regional de enfoque cualitativo que inició en julio de 2018 y se completó en marzo de 2019. La Doctora Paola Andrade, Coordinadora de Investigación de la RedLEI, lideró la investigación con la supervisión y apoyo técnico de la Doctora Rebecca Stone y la Doctora Josefina Vijil, ambas especialistas en lectoescritura inicial. Se integró un equipo de investigación regional con cinco profesionales de cada uno de los países involucrados -Guatemala, El Salvador, Honduras, Nicaragua y Costa Rica.

Los equipos de asesoría en LEI y de investigación revisaron la literatura para identificar la evidencia más reciente sobre lo que los docentes deben saber y poder hacer para la instrucción de la lectoescritura inicial. A partir de esta revisión construyeron el marco conceptual que se expuso en el apartado anterior. Luego se diseñaron los instrumentos de recolección y análisis de datos considerando la evidencia organizada en tres Dimensiones, cada una de ellas conteniendo diferentes ámbitos de conocimientos y habilidades.

Cada integrante del equipo de investigación aplicó el diseño regional en la institución participante de su país de residencia. La recolección y análisis de datos se desarrolló mediante tres tipos de actividades: (1) mapeo de los currículos de formación inicial docente en el ámbito de LEI considerando el marco conceptual común; (2) entrevistas con docentes formadores y docentes en formación y (3) observaciones de aula. Al triangular los datos de las diferentes fuentes, el estudio ofreció una imagen de qué se enseña al futuro profesorado sobre la instrucción y aprendizaje de la LEI e información para analizar en qué medida esta formación docente se acerca o aleja de lo que la evidencia investigativa sugiere.

\subsection{Unidades de análisis}

Las organizaciones miembros de RedLEI, considerando la relevancia del estudio para la calidad educativa de sus países y la región, aceptaron el desafío de dar pleno acceso a sus propios programas de formación inicial docente para que sirvieran como unidades de análisis de esta investigación. Los criterios utilizados para seleccionar los currículos de la FID a analizar, los docentes en formación y formadores a entrevistar y las aulas a observar, se describen a continuación.

\subsubsection{Mapeo de los currículos de formación inicial docente en el ámbito de LEI}

Se construyó un mapa de los programas de la FID relativos a la lectoescritura inicial en cuatro organizaciones que conforman la RedLEI: la Universidad del Valle de Guatemala 
(UVG) en Guatemala, la Universidad Pedagógica Nacional Francisco Morazán (UPNFM) en Honduras, la Universidad Centroamericana "José Simeón Cañas" en El Salvador y la Universidad de Costa Rica (UCR) en Costa Rica. Los programas universitarios que se mapearon otorgan un título o diploma de nivel superior que acredita para ejercer la docencia en la educación básica del país. En el caso de Nicaragua debido a que la Universidad Centroamericana (UCA) de este país no forma docentes de educación básica, la investigación se realizó en la Escuela Normal María Mazzarello (ENMM), única institución privada de formación docente en el país a la que se tuvo acceso. Los nombres de los programas analizados son: Guatemala, Profesorado especializado en educación primaria; El Salvador, Licenciatura en Educación Básica para primer y segundo ciclo; Honduras, Profesorado de Educación Básica para el I y II Ciclo en el Grado de Licenciatura; Nicaragua, Programa de formación inicial docente de educación primaria; y Costa Rica, Bachillerato y Licenciatura en Educación Primaria. Las características de los programas se muestran en la Tabla 1.

Tabla 1

Características de los programa de formación inicial docente

\begin{tabular}{|c|c|c|c|c|c|}
\hline & Título que otorga & Créditos & $\begin{array}{l}\text { Total } \\
\text { de } \\
\text { cursos }\end{array}$ & $\begin{array}{l}\text { Cursos } \\
\text { relacionados } \\
\text { con la LEI }\end{array}$ & $\begin{array}{l}\text { Duración de } \\
\text { la carrera } \\
\text { (años) }\end{array}$ \\
\hline Guatemala $^{\mathrm{P}, \mathrm{S}}$ & $\begin{array}{lrr}\text { Profesor } & \text { o } & \text { Profesora } \\
\text { especializada } & \text { en } \\
\text { primaria } & \end{array}$ & 78 & 32 & 9 & 3 \\
\hline El Salvador SP, S & $\begin{array}{l}\text { Licenciado o Licenciada } \\
\text { en educación Básica } \\
\text { para primer y segundo } \\
\text { ciclo }\end{array}$ & 180 & 43 & 7 & 5 \\
\hline Honduras ${ }^{\mathrm{P}, \mathrm{D}}$ & $\begin{array}{l}\text { Licenciado o Licenciada } \\
\text { en Educación Básica } \\
\text { para el I y II ciclo }\end{array}$ & 170 & 50 & 9 & 4 \\
\hline Nicaragua P, D & $\begin{array}{l}\text { Maestro de educación } \\
\text { primaria }\end{array}$ & $\begin{array}{c}\text { No } \\
\text { definido }\end{array}$ & 48 & 8 & 3 \\
\hline Costa Rica P, D & $\begin{array}{l}\text { Bachiller (142 créditos) o } \\
\text { Licenciado o Licenciada } \\
\text { (178 créditos) en } \\
\text { Educación primaria }\end{array}$ & 178 & 58 & 11 & 6 \\
\hline
\end{tabular}

Nota. $\mathrm{P}=$ Modalidad presencial; $\mathrm{SP}=$ Modalidad Semipresencial; $\mathrm{V}=$ Modalidad virtual; $\mathrm{S}=\mathrm{Plan}$ sabatino; $\mathrm{D}=$ Plan diario.

Fuente: Elaboración propia, 2020. 


\subsubsection{Entrevistas a formadores de docentes y docentes en formación}

Se revisó la malla curricular de cada uno de los programas seleccionados y se identificaron los cursos relacionados directamente con la enseñanza de la LEI (Dimensión 2 del marco conceptual). Con base a esta información se identificó al grupo a entrevistar integrado por Docentes Formadores (DF) que imparten y Docentes en Formación (DeF) que reciben estos cursos.

El total de entrevistas realizadas se modificó debido a las diferencias de organización y calendario de la malla curricular de cada programa. La cantidad de cursos que se impartieron durante el periodo de recolección de datos (septiembre 2018-enero 2019) y, por tanto, de DF y DeF disponibles para entrevistas, varió de país a país.

Se identificaron tres grupos de personas a entrevistar según contexto y disponibilidad: (1) docentes (formadores y en formación) de cursos que se imparten durante el periodo de recolección de datos; (2) docentes formadores de cursos que no se imparten en el período de recolección de datos y; (3) docentes en formación que cursan el último año del programa de formación.

Del primer grupo se contactó a docentes de no más de tres cursos que se impartieron en el periodo de recolección de datos (septiembre 2018-enero 2019). Se entrevistó al DF encargado del curso y a un mínimo de tres DeF participantes del curso, seleccionados al azar.

Para el segundo grupo de entrevistas se identificó a docentes expertos en la enseñanza de LEI que no impartieron su curso durante el periodo de recolección de datos, pero estuvieron disponibles a ofrecer información sobre cómo forman para la instrucción de la LEI. Se revisó la malla curricular de cada institución y se solicitó entrevistas a docentes de un máximo de tres cursos que cumplieran esta característica.

Para el último grupo, se seleccionó al azar a un máximo de tres DeF por institución que cursaban el último año del programa de formación. Donde se pudo realizar estas entrevistas se obtuvo información sobre la implementación del programa y el conocimiento adquirido sobre la instrucción de la lectoescritura inicial que sirvió para la comparación con los DeF que cursaban ciclos iniciales o medios de la carrera.

En los países donde no se impartieron tres cursos relacionados con la instrucción de la LEI en el período de recolección de datos, se aumentó a cinco el número de entrevistas a docentes formadores a cargo de cursos que se ofrecieron en otros ciclos. En estas 
entrevistas también se obtuvo información sobre la implementación del programa de formación inicial docente en el ámbito de la LEl.

\subsubsection{Observaciones en aulas de formación docente}

Se obtuvo información sobre la implementación del programa en las aulas de docentes en formación siguiendo la guía de observación diseñada para el estudio. Se observaron las aulas de docentes formadores entrevistados que impartieron un curso relacionado a la enseñanza de la LEl durante el periodo de recolección de datos. Se seleccionaron un máximo de tres cursos a observar. Cada miembro del grupo investigador realizó un mínimo de tres observaciones por curso. Las fechas de las observaciones se programaron según el calendario del curso para coincidir con los temas que eran de interés para la investigación porque se relacionaron directamente con la LEI.

El proceso se condujo siguiendo los principios éticos de la investigación de seres humanos. El Comité de Ética del American Institutes for Research (AIR) revisó el protocolo de investigación y dio el aval regional para su implementación en agosto del 2018. Se solicitó un consentimiento informado verbal de cada participante antes de las entrevistas y visitas de aula. El grupo docente involucrado en la investigación recibió en todo momento un trato digno, respetuoso y profesional. La información de las personas se manejó con total confidencialidad. Los datos recabados se almacenaron en un archivo de formato digital que quedó a disposición de los miembros de la RedLEl para futuras investigaciones.

\subsection{Técnicas de recolección}

A partir de las preguntas de investigación y el marco conceptual, se diseñaron los instrumentos de recolección de datos, a saber, una guía para construir los mapas curriculares, una guía para la conducción de entrevistas semiestructurada a docentes formadores y docentes en formación, y una guía para realizar las observaciones de aula. Todas las herramientas se digitalizaron para permitir al equipo de investigación el ingreso de datos desde computadoras y minimizar los errores de entrada de información. Los instrumentos pueden consultarse en https://red-lei.org/recursos/centroamerica/. La primera versión de estas herramientas fue elaborada por el equipo asesor, luego estas se discutieron y mejoraron con el equipo de investigación en un taller regional realizado en julio del 2018. 


\subsection{Procesamiento de análisis}

En noviembre de 2018, el equipo de investigación discutió en otro taller la estrategia para el análisis de la información proveniente del mapeo de los currículos, de las entrevistas, y de las observaciones. Los miembros del equipo se capacitaron antes en el uso de la aplicación informática MAXQDA para análisis cualitativo, de manera que durante el taller ya tenían el conocimiento para usar de forma eficiente la herramienta digital de organización y análisis de datos. Cuando se finalizaron el mapeo, las entrevistas, y las observaciones de aula y se completó la digitación de todos los datos, se procedió a la elaboración de un árbol de categorías usando el marco conceptual como referente (Tabla 2). La codificación de la información recolectada se auxilió del catálogo de categorías incluidas.

Es útil mencionar que el primer árbol de categorías, propuesto por el equipo asesor para su revisión y validación con el equipo de investigación, fue modificado para agregar ámbitos complementarios a cada dimensión del marco conceptual que emergieron de la información recolectada. Se añadieron además otras categorías que se denominaron "generales" porque no se relacionaban directamente con los ámbitos de las tres dimensiones, pero ofrecían información que enriquecía el análisis para responder las preguntas de investigación (Tabla 2). Los ámbitos de la Dimensión 3 también se modificaron, como se muestra en la Tabla 2. En los Anexos 1 y 2 se puede ver la lista y descripción de los ámbitos complementarios y las categorías generales incorporados luego del trabajo de campo. 
Tabla 2

Árbol de categorías para el análisis de datos

\begin{tabular}{|c|c|c|}
\hline Dimensión 1. Desarrollo infantil & $\begin{array}{l}\text { Dimensión } 2 . \\
\text { Conocimiento de la } \\
\text { Lectoescritura }\end{array}$ & Dimensión 3. Evaluación \\
\hline $\begin{array}{l}\text { Teorías del desarrollo } \\
\text { Teorías del aprendizaje y la } \\
\text { motivación } \\
\text { Desarrollo del lenguaje } \\
\text { Fundamentos de la enseñanza- } \\
\text { aprendizaje de la lectura } \\
\text { Etapas del aprendizaje de la lectura* } \\
\text { Fundamentos de la enseñanza- } \\
\text { aprendizaje de la escritura } \\
\text { Etapas del aprendizaje de la } \\
\text { escritura* } \\
\text { Neurociencia de la LEl } \\
\text { Psicología y sociología de la } \\
\text { lectoescritura } \\
\text { Impacto del contexto en el } \\
\text { aprendizaje de la LEl } \\
\text { Bilingüismo / Transferencia del } \\
\text { aprendizaje de la lectoescritura } \\
\text { Trastornos del desarrollo de la } \\
\text { lectura y escritura }\end{array}$ & $\begin{array}{l}\text { Concepto de lo impreso } \\
\text { Conciencia fonológica } \\
\text { Principio alfabético } \\
\text { Vocabulario } \\
\text { Fluidez lectora } \\
\text { Comprensión lectora } \\
\text { Lenguaje oral } \\
\text { Conexiones entre lectura y } \\
\text { escritura } \\
\text { Grafomotricidad } \\
\text { Expresión escrita } \\
\text { Convenciones de la } \\
\text { escritura } \\
\text { Habilidades implicadas en } \\
\text { la LEl* } \\
\text { Cultura lectora* }\end{array}$ & $\begin{array}{l}\text { (Versión original) } \\
\text { Propósito y tipos de evaluación } \\
\text { Diseño de evaluaciones } \\
\text { formativas } \\
\text { Aplicación de evaluaciones } \\
\text { formativas } \\
\text { Interpretación y uso de los } \\
\text { resultados de la evaluación } \\
\text { (Versión final) } \\
\text { Propósitos de la evaluación } \\
\text { Tipos de evaluación } \\
\text { Evaluación de los aprendizajes* } \\
\text { Diseño de herramientas de } \\
\text { evaluación } \\
\text { Aplicación de herramientas de } \\
\text { evaluación } \\
\text { Interpretación y uso de los } \\
\text { resultados de la evaluación }\end{array}$ \\
\hline & Categorías generales & \\
\hline $\begin{array}{l}\text { Enfoque teórico } \\
\text { Prácticas pedagógicas } \\
\text { Medios didácticos } \\
\text { Práctica para docentes en formación } \\
\text { Supervisión y Acompañamiento } \\
\text { Formas de evaluar el aprendizaje }\end{array}$ & $\begin{array}{l}\text { Estándares nacionales } \\
\text { Acceso al currículo } \\
\text { nacional prescrito } \\
\text { Tiempo previsto para la } \\
\text { enseñanza del } \\
\text { conocimiento de la LEI }\end{array}$ & $\begin{array}{l}\text { Tiempo real dedicado a la } \\
\text { enseñanza del conocimiento de } \\
\text { la LEl } \\
\text { Bibliografía } \\
\text { Perfil del docente formador }\end{array}$ \\
\hline
\end{tabular}

Luego de completar la versión final del árbol de categorías, el equipo de investigación inició la codificación con la ayuda de la aplicación informática MAXQDA. La coordinadora del estudio regional apoyó el proceso de forma permanente mediante reuniones semanales y revisiones intermedias de la información catalogada. Al terminar la codificación, se agruparon los segmentos de textos relevantes para un análisis de tipo categorial que permitiera responder las preguntas de investigación.

Para responder la primera pregunta: ¿Cómo se alinea el currículum de formación inicial docente con la evidencia sobre cómo los niños aprenden la lectoescritura y qué deben saber y poder hacer los maestros para su instrucción efectiva?, se utilizó la información de los mapas curriculares codificados en categorías directamente relacionadas con las tres dimensiones del marco conceptual. 
La triangulación de datos de diferentes fuentes ayudó a responder tanto la segunda pregunta: ¿Cuáles son las fortalezas y las brechas del plan de estudios de formación inicial docente en relación con la evidencia investigativa?, como la tercera: ¿En qué se diferencia el currículum prescrito del currículo realmente aplicado en los centros de formación inicial docente en el ámbito de la lectoescritura inicial? Para ello se organizó y analizó la información obtenida del mapeo del currículo, las entrevistas y las observaciones considerando las categorías de las tres dimensiones del marco conceptual y las categorías generales.

En marzo del 2019, al finalizar la etapa de codificación y análisis de datos, se realizó un taller regional para discutir los hallazgos y definir los criterios para la producción de los informes de la investigación conducida en cada institución participante. En esta actividad se proporcionaron herramientas para ayudar a cada miembro del equipo investigador en la redacción de su documento final. Los resultados particulares de la investigación se continuaron discutiendo durante dos meses en sesiones individuales. Los miembros del equipo recibieron retroalimentación de los productos entregados y pautas para la edición del informe de investigación para su posterior publicación.

\section{Resultados}

Cada miembro del equipo investigador produjo un artículo para describir los hallazgos y análisis de la FID en el ámbito de la LEI en la institución asignada. Los artículos, presentados en esta revista, informan qué saberes y competencias se incluyen en la preparación del futuro cuerpo docente para la instrucción de la lectoescritura inicial, y los comparan con los saberes y competencias que la evidencia investigativa señala como necesarios. El resultado de la comparación es una evaluación objetiva de las fortalezas y debilidades de los programas de formación docente participantes en la investigación. Los hallazgos sobre las brechas entre lo que se enseña y lo que la evidencia sugiere enseñar, se traducen en recomendaciones para la reorganización de los programas de la FID y su alineación con la evidencia investigativa sobre la instrucción efectiva de la LEI. A través de estos artículos, la investigación promovida por la RedLEI espera contribuir a llenar una de los tantos vacíos de conocimiento sobre los factores que inciden en la calidad de la instrucción y aprendizaje de la lectoescritura inicial. 
Puede acceder a los resultados por país en cada uno de los siguientes enlaces.

\section{GUATEMALA}

\section{EL SALVADOR}

\section{HONDURAS}

NICARAGUA

\section{COSTA RICA}

\section{Conclusiones}

La premisa teórica de esta investigación regional proviene del conocimiento investigativo según el cual hay una relación estrecha entre el dominio de determinados conocimientos y habilidades, y la instrucción efectiva de la lectoescritura inicial. Se adoptó como perspectiva que la formación del cuerpo docente es el factor clave de la calidad de la enseñanza de la LEI y, por tanto, es fundamental asegurarse que éste se prepare como especialista en esta materia.

Los hallazgos encontrados en los programas de formación docente de cinco países de Centroamérica coinciden con investigaciones previas (Kyeyune et al., 2011; Louzano y Moriconi 2014; OREALC-UNESCO, 2013) y muestran un panorama preocupante por las numerosas brechas identificadas entre cómo se educa al futuro magisterio y lo que la evidencia sugiere. El estudio revela que la formación inicial que reciben muchos docentes en formación es insuficiente para que enfrenten los retos del trabajo en el aula y apliquen metodologías efectivas para la instrucción de la LEI. Los vacíos de la formación que esta investigación identificó limitan la capacidad del futuro docente de abordar las necesidades particulares de la niñez con la que deberá trabajará.

El cuerpo docente en formación aprende de las teorías del desarrollo infantil a un nivel conceptual o teórico, sin llegar a comprender la relación entre los cambios biológicos, emocionales y socio-ambientales que ocurren durante la niñez y el aprendizaje de la LEI. Desconoce, por tanto, cómo diseñar propuestas pedagógicas efectivas y motivadoras para el alumnado. La formación docente deja un gran vacío sobre la instrucción en contextos multilingües a pesar de su gran importancia para la región (Akyeampong et al., 2011). Este vacío se suma a otras brechas de conocimiento sobre la instrucción de la LEI en poblaciones vulnerables.

Los programas de la FID en Centroamérica no están aprovechando la abundante evidencia investigativa sobre cómo se fomentan las habilidades que la niñez necesita 
desarrollar para aprender a leer y escribir efectivamente. Aunque existe conciencia de la necesidad del modelaje en el aula de formación inicial y de la práctica supervisada por un especialista del área, la mayoría de las veces las prácticas no se implementan como se prescriben. Incluso, cuando el plan de estudios incluye habilidades clave para el desarrollo de la LEI, rara vez se especifica cómo enseñar esas habilidades, dejando este contenido a nivel pedagógico y generalista (Kyeyune et al., 2011; Louzano y Moriconi 2014; OREALCUNESCO, 2013). También es preocupante el hallazgo de que no existe un marco conceptual que guíe el currículo; se confunde el enfoque con las metodologías e inclusive con actividades aisladas que no se sustentan en una teoría.

La evaluación se reduce a la medición del desempeño del alumnado. Al futuro cuerpo docente no se le prepara para evaluar el aprendizaje de la LEI y utilizar los resultados de las evaluaciones para tomar decisiones relativas al trabajo en el aula, o para guiar la actualización y mejora de la instrucción.

En términos generales, existe solo una alineación parcial de los programas a la evidencia en las diversas dimensiones analizadas. En la mayoría de los ámbitos se enseñan fundamentos teóricos que no se traducen a la práctica, ni se vinculan a las particularidades epistemológicas de le lectoescritura inicial. Se trata entonces de una educación generalista, con poca aplicación concreta a la enseñanza. Se identifican entre las mayores carencias la escasa oportunidad de práctica supervisada, y la reducida formación para el diseño, selección y uso de recursos para la instrucción y evaluación del aprendizaje de la LEI en el contexto particular de los países.

La formación de docentes especializados en la enseñanza de la LEI requiere de docentes formadores de nivel universitario con experiencia y conocimiento en esta materia. Los hallazgos de la investigación sugieren una brecha también en la formación de formadores. Frente a muchos de los grupos que estudian magisterio se encuentra un cuerpo de docentes formadores que también carece de los saberes y habilidades que deberían enseñar sobre la LEI. Esta brecha podría explicar el escaso seguimiento del plan de estudios prescrito, que conduce a que éste no se imparta por completo y a que las prácticas pedagógicas sugeridas no se lleven a cabo.

Los hallazgos de esta investigación regional confirman lo que señalan otros estudios: quienes se están preparando para la docencia y se van a encargar de la instrucción de la lectoescritura en los primeros grados en Centroamérica, no están recibiendo la formación para acompañar a la niñez en la importante tarea de aprender a leer y escribir (Bruns y 
Luque, 2015; Kyeyune et al., 2011; Louzano y Moriconi 2014; LRCP, 2016; OREALCUNESCO, 2013). Los programas de FID analizados no están alineados en su totalidad con la evidencia sobre los procesos y factores involucrados en la efectividad del aprendizaje de la LEI.

La RedLEI reconoce que no es posible generalizar sobre la formación docente inicial en LEI a partir de las conclusiones de una sola investigación. Este estudio regional se propone, por tanto, como el primero de una línea de investigación. El conocimiento acumulado debe ampliarse y profundizarse en estudios del mismo tema que consideren otras perspectivas y metodologías e involucren a más actores clave del sector educativo.

Finalmente, los hallazgos de esta investigación han sido formulados para promover la discusión, a lo interno de las instituciones participantes, sobre los ajustes que sus programas de estudio deberían experimentar. RedLEl se propone apoyar a sus organizaciones miembros para que se reduzca la brecha entre cómo forman al futuro magisterio en el ámbito de la LEI y lo que la evidencia científica sugiere. También se propone incidir en la mejora de las políticas de formación docente inicial a nivel de país y región.

\section{Agradecimientos}

La RedLEI agradece a las autoridades de las universidades y centros participantes por responder nuestras preguntas y permitirnos generosamente tener acceso a sus aulas, estudiantes y programas de estudio. Estamos en deuda con la Doctora Rebecca Stone y la Doctora Josefina Vijil, especialistas en lectoescritura inicial del Programa de Capacidades LAC Reads (PCLR), quienes asesoraron el proyecto desde su formulación hasta la producción de los informes finales. Agradecemos a la Doctora Ana Patricia Elvir, visionaria y apasionada del tema, por su acompañamiento a lo largo de todo el proceso. Los equipos técnicos del PCLR y de la RedLEl nos ofrecieron apoyo organizativo, logístico y administrativo que resultó fundamental para cumplir las tareas de este proyecto. Nuestro especial agradecimiento al grupo fundador de RedLEl y a la Agencia de los Estados Unidos para el Desarrollo Internacional (USAID) por su patrocinio y por la confianza depositada en este equipo para producir esta investigación, la primera que aborda esta temática para ofrecer evidencia regional que guíe la toma de decisiones para mejorar la calidad educativa de la región. 


\section{Referencias}

Adger, Carolyn Temple, Snow, Catherine E., y Christina, Donna (2018). What Teachers Need to Know About Language. Washington, DC: CAL Series on Language Education.

Akyeampong, Kwame, Pryor, John y Ampiah Joseph Ghartey. (2006). A vision of successful schooling: Ghanaian teachers" understandings of learning, teaching and assessment. Comparative Education, 42, 155-176. doi: https://doi.org/10.1080/03050060600627936

Akyeampong, Kwame, Pryor, John, Westbrook, Jo y Lussier, Kattie (2011). Teacher preparation and continuing professional development in Africa: Learning to teach early reading and mathematics. England: Center for International Education, University of Sussex.

Alamargot, Denis y Chanquoy, Lucille. (2001). Through the models of writing. DordrechtBoston-London: Kluwer Academic Publishers.

Alviz, Leire (2012). La grafomotricidad en Educación Infantil. Revista Arista Digital, 16(6), 4854.

American Psychiatric Association (2014). Guía de consulta de los criterios diagnósticos del DSM-5: Spanish Edition of the Desk Reference to the Diagnostic Criteria from DSM-5®. Washington D.C.: American Psychiatric Pub.

American Speech-Language-Hearing Association (2008). Roles and Responsibilities of Speech-Language Pathologists in Early Intervention: Guidelines [Guidelines]. Recuperado de www.asha.org/policy

Asociación Nacional de Universidades e Instituciones de Educación Superior (1997). La Evaluación y Acreditación de la Educación Superior en México. Revista de la Educación Superior, 101, 1-19. Recuperado de http://publicaciones.anuies.mx/pdfs/revista/Revista101_S3A2ES.pdf

August, Diane y Shanahan, Timothy. (Eds.). (2006). Developing literacy in second-language learners: Report of the National Literacy Panel on Language-Minority Children and Youth. Mahwah, NJ: Lawrence Erlbaum.

Barletta Manjarrés, Norma, Cortez Román, Nolvia A. y Medzerian, Star. (2012). From official educational policy to the composition classroom: Reproduction through metaphor and metonymy. Journal of Writing Research, 4(1), 31-51. http://dx.doi.org/10.17239/jowr2012.0401 .2

Bazerman, Charles, Farmer, Frank, Halasek, Kay y Williams, Joseph M. (2005). Responses to Bakhtin's "Dialogic Origins and Dialogic Pedagogy of Grammar: Stylistics as Part of Russian Language Instruction in Secondary Schools" Further Responses and a Tentative Conclusion. Written Communication, 22(3), 363-374.

Bellei, Cristián, Contreras, Daniel y Valenzuela, Juan Pablo. (2010). Ecos de la revolución pingüina. Santiago, Chile: UNICEF. 
Berman, Ruth A. (2004). Between emergence and mastery: The long developmental route of language acquisition. En R.A. Berman (Ed.). Language development across childhood and adolescence. Amsterdam: John Benjamins.

Berninger, Virginia W. y Amtmann, Dagmar. (2003). Preventing written expression disabilities through early and continuing assessment and intervention for handwriting and/or spelling problems; Research into practice. En H. L. Swanson, K. R. Harris, y S. Graham (Eds.), Handbook of learning disabilities (pp. 345-363). New York, Estados Unidos: Guilford Press.

Bezemer, Jeff y Gunther Kress (2010). Multimodality: A social semiotic approach to contemporary communication. Londrés: Routledge.

Bialystok, Ellen. (2011). Reshaping the mind: the benefits of bilingualism. Canadian Journal of Experimental Psychology/Revue canadienne de psychologie expérimentale, 65(4), 229. Recuperado de https://www.ncbi.nlm.nih.gov/pmc/articles/PMC4341987/

Brem, Silvia, Bach, Silvia, Kucian, Karin, Kujala, Janne V., Guttorm, Tomi K., Martin, Ernst, Lyytinen, Heikki, Brandeis, Daniel y Richardson, Ulla. (2010). Brain sensitivity to print emerges when children learn letter-speech sound correspondences. Proceedings of the National Academy of Sciences, 107(17), 7939-7944.

Bruning, Roger y Kauffman, Douglas (2015). Self-efficacy beliefs and motivation in writing development. En C. A. MacArthur, S. Graham, y J. Fitzgerald (Eds.), Handbook of Writing Research (2 Ed., pp. 160-173). Nueva York, Estados Unidos: Guilford Press.

Bruns, Bárbara y Luque, Javier. (2015). Profesores Excelentes: Cómo mejorar el aprendizaje en América Latina y el Caribe. Washington, Estados Unidos: Grupo del Banco Mundial.

Bui, Yvonne N., Schumaker, Jean B. y Deshler, Donald D. (2006). The effects of a strategic writing program for students with and without learning disabilities in inclusive fifth-grade classes. Learning Disabilities Research and Practice, 21(4), 244-260. doi:10.1111/j.1540-5826.2006.00221.x

Camargo, Geraldina, Montenegro, Raquel, Maldonado, Sophia y Magzul, Justo. (2013). Aprendizaje de la lectoescritura. Guatemala: USAID/Reforma Educativa en el Aula.

Casey, B.J, Davidson, Matthew. y Rosen, Bruce. (2002) Functional magnetic resonance imaging: basic principles of and application to developmental science. Developmental Science, 5(3), 301-309.

Cervantes, Gustavo y Colmenero, Igna. (2016). Sobre el diseño de medios didácticos digitalizados. Cuba: Maestro y sociedad.

Chall, J. S. (1983). Stages of Reading Development. New York: McGraw-Hill.

Chappuis, Stephen y Chappuis, Jan. (2008). The Best Value in Formative Assessment. Educational Leadership, 65(4), 14-19. 
Chesterfield, Ray y Abreu-Combs, Adriana. (2011). Center for excellence in teacher training (CETT) two-year impact study report. USAID Bureau for Latin America and Caribbean.

CIEN e Inter- American Dialogue (2015). GUATEMALA: El Estado de las Políticas Públicas docentes. Guatemala: Autor.

Condemarín, Mabel y Medina, Alejandra. (2000). El diario en el aula. Santiago, Chile: LOM.

Copple, Carol y Bredekamp, Sue. (2009). Developmentally appropriate practice in early childhood programs serving children from birth through age Eight. Washington, DC, Estados Unidos: National Association for the Education of Young Children (NAEYC).

Cunningham, Anne, Perry, Kathin, Stanovich, Keith y Stanovich, Paula. (2004). Disciplinary knowledge of $\mathrm{K}-3$ teachers and their knowledge calibration in the domain of early literacy. Annals of Dyslexia, 54, 139-167. doi:10.1007/s11881-004-0007-y

Darling-Hammond, Linda y Bransford, John. (2005). Preparing teachers for a changing world: What teachers should learn and be able to do. San Francisco: Jossey-Bass.

Dembélé, Martial and Lefoka, Pulane. (2007). Pedagogical renewal for quality universal primary education: Overview of trends in Sub-Saharan Africa, International Review of Education, 53, 531-553. doi: 10.1007/s11159-007-9066-8

Diamond, Julie. (1996). Status and power in verbal interaction: a study of discourse in a close-knit social network. Amsterdam: Pragmatics and Beyond New Series.

Díaz, Carmen, Villalón, Malva y Adlerstein, Cynthia. (2015). Conocimiento práctico para la enseñanza del lenguaje oral y escrito de estudiantes de educación inicial: Una aproximación desde la elaboración de mapas conceptuales. Interdisciplinaria, 32(2), p 331-345

Duque, Paula Andrea., Rodríguez, Juan Carlos y Vallejo, Sandra Lorena. (2013). Prácticas Pedagógicas y su relación con el Desempeño Académico. Colombia: Universidad de Manizales.

Englert, Carol Sue, Garmon, Art, Mariage, Troy, Rozendal, Mary, Tarrant, Kathi y Urba, Joyce. (1995). The early literacy project: Connecting across the literacy curriculum. Learning Disability Quarterly, 18(4), 253-275. doi.org/10.2307/1511233

Ferreiro, Emilia. y Gómez, Margarita. (1986). Nuevas perspectivas sobre los procesos de lectura y escritura. Buenos Aires, Argentina: Siglo XXI.

Ferreiro, Emilia. y Teberosky, Ana. (1979). Los sistemas de escritura en el desarrollo del niño. México: Siglo XXI editores.

Fondo de las Naciones Unidas para la Infancia. (2012). Development of a Teacher Education Strategy Framework Linked to Pre and In-Service Teacher Training in Myanmar. Myanmar: Author. 
Gallego, José Luis., García, Antonio y Rodríguez, Antonio. (2014). Estrategias metacognitivas en la expresión escrita: un estudio de caso con futuros docentes. Bordón, 66(4), 39-53. doi: 10.13042/Bordon.2014.66404

Genesee, Fred, Lindholm-Leary, Kathryn, Saunders, William y Christian, Donna. (2006). Educating English language learners. New York, Estados Unidos: Cambridge University Press.

Goldman-Rakic, Patricia S. (1987). Development of Cortical Circuitry and Cognitive Function. Child Development, 58, 601-22. http://dx.doi.org/10.2307/1130201

Gove, A., Chabbott, C., Dick, A. DeStefano, J., King, S., Mejia, J. and Piper, B. (2015). Early learning assessments: a retrospective. Background Paper for Education for All Global Monitoring Report 2015. Paris, France: UNESCO.

Graham, Steve y Hebert, Michael A. (2010). Writing to read: Evidence for how writing can improve reading. A Carnegie Corporation Time to Act Report. Washington, DC, United States: Alliance for Excellent Education.

Greene, William H. (1999). Análisis econométrico. Madrid, España: Prentice Hall.

Hayes, John R. (1996). A new framework for understanding cognition and affect in writing. In C. M. Levy, y S. Ransdell (Eds.), The science of writing: Theories, methods, individual differences and applications (pp. 1-27). Mahwah, NJ, United States: Lawrence Erlbaum Associates.

Hayes, John R. y Flower, Linda S. (1980). Identifying the organization of writing processes. In L. Gregg y E. R. Steinberg (Eds.), Cognitive processes in writing (pp. 3-30). Hillsdale, $\mathrm{NJ}$ : Lawrence Erlbaum Associates.

Holdgreve-Resendez, Richard. (2010). Concepts of Print (cont.). Extracted from Concepts of Print and Genre Slides from angel.msu.edu on September 22, 2010. TE 301: Learners and Learning in Context.

Hoover, Wesley A., y Gough, Phillip B. (1990). The simple view of reading. Reading and Writing: An Interdisciplinary Journal, 2, 127-160. doi:10.1007/BF00401799.

Hoyle, Susan M. y Adger, Carolyn Temple. (1998). Introduction. En S.M. Hoyle y C.T. Adger (eds) Kids Talk: Strategic Language Use in Later Childhood (pp. 3- 22). New York, United States: Oxford University Press.

International Literacy Association. (2010). Standards for Reading Professionals - revised 2010. A position statement of the International Reading. Newark, DE, United States: Author.

International Reading Association. (2007). Teaching Reading Well. A Synthesis of the International Reading Association's Research on Teacher Preparation for Reading Instruction. Newark, DE, United States: author. 
Kyeyune, Robinah, Mirembe, Robinah, Baleeta, Margaret, Sentongo, John, Nambi, Rebecca, Katende, George, ... Lussier, Kattie (2011). Teacher preparation and continuing professional development in Africa (TPA): Learning to teach reading and mathematics and its influence on practice in Uganda. Uganda: University of Sussex Centre for International Education.

LAC Reads Capacity Program. (2016). Early Grade Reading in Latin America and the Caribbean: A Systematic Review. USAID

Leppänen, Paavo H. T., Hämäläinen, Jarmo A., Salminen, Hannek, Eklund, Kenneth M., Guttorm, Tomi K., Lohvansuu, Kaisa, ... Lyytinen, Heikki. (2010). Newborn brain eventrelated potentials revealing atypical processing of sound frequency and the subsequent association with later literacy skills in children with familial dyslexia. Cortex, 46(10), 1362-1376. doi:10.1016/j.cortex .2010.06.003

Liston, Conor, Watts, Richard, Tottenham, Nim, Davidson, Matthew C., Niogi, Sumit, Ulug, Aziz M. y Casey, B. J. (2005). Frontostriatal microstructure modulates efficient recruitment of cognitive control. Cerebral Cortex, 16(4), 553-560.

Litt, Deborah G., Martin, Susun D. y Place, Nancy A. (2015). Literacy Teacher Education: Principles and Effective Practices. Nueva York, United States: Guilford Publications.

Louzano, Paula, y Moriconi, Gabriela. (2014). Visión de la docencia y características de los sistemas de formación docente en Finlandia, Singapur y Estados Unidos. Cadernos Cenpec, 4(2), 30-53. Recuperado de http://www.cadernos.cenpec.org.br/cadernos/index.php/cadernos/article/viewFile/287/2 85

Lucas, Tamara y Grinberg, Jaime. (2008). Responding to the linguistic reality of mainstream classrooms: Preparing all teachers to teach English language learners. En Cochran Smith, M., Feiman-Nemser, S., y Mclntyre J. (Eds.), Handbook of research on teacher education: Enduring issues in changing contexts (pp. 606- 636), Nueva Jersey, Estados Unidos: Lawrence Erlbaum.

Manghi, Dominique (2011). La perspectiva multimodal sobre la comunicación. Desafíos y aportes para la enseñanza en el aula. Diálogos Educativos, 21(22), 4-15. Recuperado de https://dialnet.unirioja.es/servlet/articulo?codigo=3931351

McEwan, Patrick. (2014). Improving learning in primary schools of developing countries: A meta-analysis of randomized experiments. Review of Educational Research, 85(3)142. https://doi.org/10.3102/0034654314553127

McField, Grace y McField, David R. (2014). The consistent outcome of bilingual education programs: A meta-analysis of meta-analyses. In G. P. McField (Ed.), The miseducation of English learners (pp. 267-298). Charlotte, NC, United States: Information Age. 
McLaren, Donald G., Ries, Michele, L., Xu, Guofan, y Johnson, Sterling C. (2012). A Generalized Form of Context-Dependent Psychophysiological Interactions (gPPl): $A$ Comparison to Standard Approaches. Recuperado de https://www.ncbi.nlm.nih.gov/pmc/articles/PMC3376181/

Menyuk, Paula y Brisk, Maria (2005). Language Development and Education: Children with Varying Language Experiences Paperback. Springer.

Moats, Louisa. (2009). Knowledge foundations for teaching reading and spelling. Read Writ, 22, 379-399. doi: 10.1007/s11145-009-9162-1

Moon, Bob, Leach, Jenny y Stevens, Mary-Priscilla. (2005). Designing open and distance learning for teacher education in Sub-Saharan Africa: A toolkit for educators and planners. Washington DC, United States: The World Bank.

Munakata, Yuko y Yerys, Benjamin E. (2001). All together now: When dissociations between knowledge and action disappear. Psychological Science, 12(4), 335-337. Recuperado de https://journals.sagepub.com/doi/10.1111/1467-9280.00361

National Institute of Child Health and Human Development. (2000). Report of the National Reading Panel. Teaching children to read: An evidence-based assessment of the scientific research literature on reading and its implications for reading instruction: Reports of the subgroups (NIH Publication No. 00-4754). Washington, DC: U.S. Government Printing Office. Recuperado de http://www.nichd.nih.gov/ publications/nrp/report.htm

National Reading Panel, National Institute of Child Health, \& Human Development. (2000). Report of the National Reading Panel: Teaching children to read: An evidencebased assessment of the scientific research literature on reading and its implications for reading instruction: Reports of the subgroups. Recuperado de https://www.nichd.nih.gov/sites/default/files/publications/pubs/nrp/Documents/report.pdf

National Research Council. (2010). Preparing Teachers: Building Evidence for Sound Policy. Washington, DC, United States: The National Academies Press. https://doi.org/10.17226/12882.

Olesen, Pernille J, Westerberg, Helena y Klingberg, Torkel. (2004). Increased prefrontal and parietal activity after training of working memory. Nature Neuroscience, 7(1), 75. doi:10.1038/nn1165

Oficina Regional de Educación para América Latina y el Caribe y Organización de las Naciones Unidas para la Educación, la Ciencia y la Cultura. (2013). Situación Educativa del América Latina y el Caribe: Hacia la educación de calidad para todos al 2015. Santiago, Chile: Ediciones del Imbuche.

Organización de las Naciones Unidas para la Educación, la Ciencia y la Cultura. (2012). EFA Global Monitoring Report 2012: Youth and Skills: Putting education to work. Paris, France: Author. 
Organización de las Naciones Unidas para la Educación, la Ciencia y la Cultura. (2016). Education for people and planet: creating sustainable futures for all, Global Education Monitoring Report, 2016. Paris, France: Author.

Ortega, Lourdes (2008). Understanding second language acquisition. Londres: Hodder Education.

Patiño, Gustavo (2005). Citas y referencias bibliográficas. Bogotá, Colombia: Pontificia Universidad Javeriana.

Pontefract, Caroline y Hardman, Frank. (2005) The discourse of classroom interaction in Kenyan primary schools. Comparative Education, 42(1), 87-106. Recuperado de http://www.jstor.org/stable/30044520

Rivkin, Steven, Hanushek, Erick, y Kain, John. (2005). Teachers, schools and academic achievement. Econometrica, 73, 417-458.

Rolstad, Kellie, Mahoney, Kate y Glass, Gene V. (2005). The big picture: a meta-analysis of program effectiveness research on English Language Learners. Educational Policy, 19(4), 572-594. doi:10.1177/0895904805278067

Romaine, Suzanne. (1995). Bilingualism (2 ${ }^{\text {nd }}$ Ed.). Oxford, U.K: Blackwell Publishers Inc.

Sayago, Zorayda Beatriz., y Chacón, María Auxiliadora. (2006). Las prácticas profesionales en la formación docente: hacia un nuevo diario de ruta. Mérida, Venezuela. Educere, 10(32), 55-66.

Shanahan, Timothy. (2015). Common Core State Standards: A New Role for Writing. Elementary School Journal, 115, 464-479.

Sitko, Barbara M. (1998). Knowing how to write: metacognition in writing instruction. En D. J. Hacker, J. Dunlosky y A. C. Graesser (eds.), Metacognition in Educational Theory and Practice (pp. 93-116). London: Lawrence Erlbaum Associates.

Stahl, Katherin A., Flanigan, Kevin y McKenna, Michael C. (2009). Assessment for reading instruction, second edition. Nueva York, United States: Guilford.

Tantaleán Odar, Luis Reynaldo, Vargas, Mariela Janeth y López, Oscar. (2016). El Monitoreo pedagógico en el desempeño profesional docente. Perú: Didáctica, Innovación y Multimedia (DIM).

Teberosky, Ana. (2000). Relectura de los sistemas de escritura en el desarrollo del niño. En F. Avendaño y M. Baez (Comps.), Sistemas de escritura, constructivismo y educación (pp. 24-42). Argentina: Homo Sapiens.

Teacher Education and Development Study in Mathematics. 2008. [Base de datos]. Amsterdam: Author. Recuperado de http://www.iea.nl/teds-m.html 
Tunmer, William E. y Hoover, Wesley A. (2019). The cognitive foundations of learning to read: a framework for preventing and remediating reading difficulties. Australian Journal of Learning Difficulties, 24(1), 75-93.

Witte, Stephen P. y Faigley, Lester. (1981). Coherence, cohesion, and writing quality. College Composition and Communication, 32(2), 2-11.

Wong-Fillmore, L. y Snow, Catherine E. (2000). What teachers need to know about language. Washington, DC, United States: ERIC Clearinghouse on Languages and Linguistics. 


\section{Anexos}

\subsection{Anexo 1. Descripción de ámbitos complementarios derivados de la información recolectada}

\subsubsection{Etapas del aprendizaje de la lectura}

Es el conocimiento de que la comprensión del lenguaje escrito es un proceso gradual de aprendizaje que ocurre en etapas definidas.

\subsubsection{Etapas del aprendizaje de la escritura}

Es el conocimiento de que la escritura, que expresa hechos, pensamientos y sentimientos mediante signos gráficos, se aprende en un proceso gradual con etapas definidas

\subsubsection{Habilidades implicadas en la LEI}

Es el conocimiento de todas las habilidades involucradas en la adquisición y desarrollo de la lectoescritura inicial.

\subsubsection{Cultura lectora}

Son las prácticas relacionadas con la promoción de la lectura habitual y regular de libros, materiales de información impresos o digitales en la búsqueda de conocimiento, información o entretenimiento a través de la palabra escrita.

\subsubsection{Evaluación de los aprendizajes}

Es el conocimiento del proceso continuo, integral y participativo que permite identificar, analizar y explicar una problemática mediante información relevante y proporciona, como resultado, conclusiones que sustentan la consecuente toma de decisiones (Asociación Nacional de Universidades e Instituciones de Educación Superior, 1997).

\subsection{Anexo 2 Definición de categorías generales}

\subsubsection{Enfoque teórico}

Referente teórico con que se analiza la lectoescritura inicial y se interpreta el proceso de su instrucción y aprendizaje.

\subsubsection{Prácticas pedagógicas}

Se denomina así a las acciones que hace el cuerpo docente para favorecer la formación integral del estudiantado tales como enseñar, comunicar, socializar experiencias, reflexionar desde la cotidianidad, y evaluar los procesos cognitivos y la forma de relacionarse con la comunidad educativa (Duque, Rodríguez, Vallejo, 2013). 


\subsubsection{Medios didácticos}

Son los recursos mediadores del aprendizaje que contribuyen al logro de los objetivos de un contenido dentro del proceso enseñanza aprendizaje (Cervantes \& Colmenero, 2016).

\subsubsection{Prácticas para docentes en formación}

Son las actividades que se organizan para colocar al docente en formación en situaciones reales en las que observa, interviene, reflexiona, reconstruye, valora, desarrolla habilidades para la solución de problemas y reafirma su compromiso social y ético, con la intención de ir construyendo su identidad como docente (Sayago y Chacón, 2006).

\subsection{Supervisión y acompañamiento}

Es el monitoreo pedagógico con el cual se da seguimiento permanente a las tareas asignadas al docente en formación o docente formador, con el objetivo de conocer el nivel de su desempeño para asesorarlo y capacitarlo según sus resultados (Tantaleán Odar, Vargas y López 2016).

\subsubsection{Formas de evaluar el aprendizaje}

Son los medios para obtener evidencias que ayuden a determinar el grado de logro alcanzado de los objetivos de aprendizaje, e implementar acciones de mejora.

\subsubsection{Estándares nacionales}

Son las metas de calidad en los diferentes ámbitos educativos a partir de las cuales se mide el conocimiento estudiantil adquirido y el desempeño del cuerpo docente, directivo y administrativo del sistema escolar (Bellei, Contreras, y Valenzuela, 2010).

\subsubsection{Acceso al currículo nacional prescrito}

Es la existencia o ausencia de oportunidades para conocer el currículo nacional o documento que describe las capacidades, competencias, conceptos, destrezas, habilidades y actitudes que debe lograr el alumnado en cada nivel, ciclo o modalidad del sistema educativo.

\subsubsection{Tiempo previsto para la enseñanza del conocimiento de la LEI}

Es el número de horas asignadas a la enseñanza del conocimiento de la LEI en los programas de los cursos analizados.

\subsubsection{Tiempo real dedicado a la enseñanza del conocimiento de la LEI}

Es el número de horas que el cuerpo docente formador y cuerpo docente en formación informan, durante las entrevistas, que se dedicaron a la enseñanza y aprendizaje del conocimiento de la LEI durante las observaciones y las entrevistas. 


\subsubsection{Bibliografía}

Son las fuentes de consulta y búsqueda de las que dispone el cuerpo docente formador y en formación. Incluyen libros, revistas, periódicos, entrevistas, documentos, películas, emisiones de radio o televisión, información de internet, discos multimedia, avisos publicitarios y otros medios similares de divulgación del conocimiento (Patiño, 2005).

\subsubsection{Perfil del docente formador}

Es el conjunto de competencias y habilidades que cada institución educativa establece como requisitos para que un profesional ejerza el cargo de docente formador. Incluye criterios valorativos e indicadores de calidad del desempeño. 
Revista indizada en
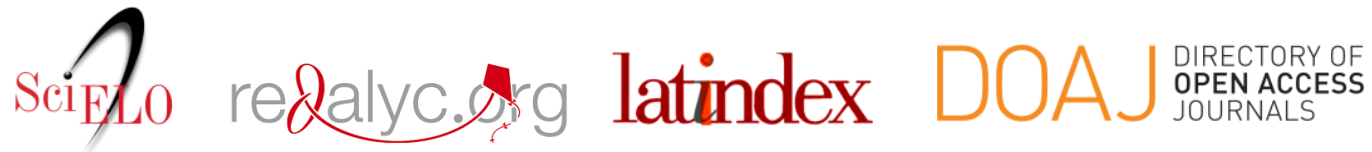

Distribuida en las bases de datos:

๑ Dialnet

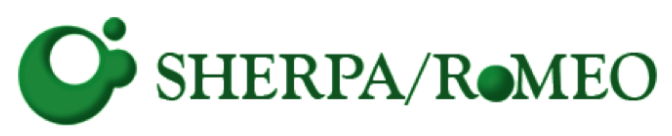

REDIB

Red Iberoamericana

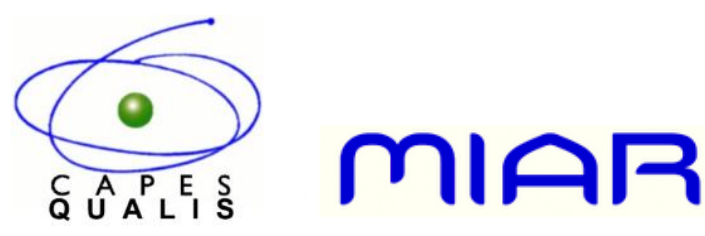

